\title{
Transmission characteristics of SMN from 227 spinal muscular atrophy core families in China
}

\author{
Yanyan Cao ${ }^{1} \cdot$ Yujin $\mathrm{Qu}^{1} \cdot$ Jinli Bai ${ }^{1} \cdot$ Miaomiao Cheng ${ }^{1} \cdot$ Yuwei Jin ${ }^{1} \cdot$ Hong Wang ${ }^{1} \cdot$ Fang Song $^{1}$
}

Received: 23 November 2019 / Revised: 5 January 2020 / Accepted: 26 January 2020 / Published online: 13 February 2020

(c) The Author(s), under exclusive licence to The Japan Society of Human Genetics 2020

\begin{abstract}
To define the relationship between the survival motor neuron 1 gene (SMN1) and SMN2, and explore the variability of these two genes within the generations, SMN1 and SMN2 copy numbers were determined for 227 SMA families. The association analysis indicated that there was a negative correlation between the copy number of SMN1 and SMN2 (Spearman $=-0.472$, $P<0.001)$ in 227 SMA children and 454 of their parents. The average $S M N$ copies from father and mother in each SMA family were used to represent the copy number in the parent's generation. Subsequently, $S M N$ transmission analysis showed that the similar distribution trend of SMN1 and SMN2 copy number was not only in the SMA children and their parents' generation but also in the non-SMA families. Moreover, when the SMN2 copy number was one in the parent's generation, $75 \%$ of their SMA children had type I and $25 \%$ of them had type II/III. However, when the SMN2 copies were three in the parent's generation, all of their SMA children were type II/III. Therefore, the diversity of SMN copies was mostly inherited and the SMN2 copy number in the parent's generation could predict the disease severity of SMA children to some extent.
\end{abstract}

\section{Introduction}

Spinal muscular atrophy (SMA) is caused by deficiency of the survival motor neuron (SMN) protein, resulting in degeneration of anterior horn cells of the spinal cord and characterized by symmetrically proximal muscle weakness and atrophy of lower limbs, and can involve multiple systems. SMA is a leading genetic cause of infant mortality, with an estimated incidence of 1/10 000 live births and a carrier frequency from $1 / 35$ to $1 / 60$ worldwide $[1,2]$. Based on age at onset and the highest level of motor function achieved, childhood SMA can be classified into three groups: severe type I (MIM 253300), intermediate type II (MIM 253550), and mild type III (MIM 253400).

Survival motor neuron 1 gene $(S M N I)$ is the diseasecausing gene, and another nearly identical gene, $S M N 2$, is the

Supplementary information The online version of this article (https:// doi.org/10.1038/s10038-020-0730-1) contains supplementary material, which is available to authorized users.

Fang Song

songf_558@263.net

1 Department of Medical Genetics, Capital Institute of Pediatrics, Beijing, China phenotype-modified gene in a copy-dependent manner. Most often, SMA is inherited from carrier parents in an autosomal recessive pattern. However, $\sim 2 \%$ of cases result from de novo variant [3]. In SMA patients, about $95 \%$ of cases carry a homozygous loss of SMN1, caused by gene deletion or conversion of SMN1 to $S M N 2$. And the remaining 5\% are compound heterozygous variant in SMN1 [4].

SMA-related genes are located on chromosome 5q13, a complex genomic structure region, which is predisposed to gene duplication, deletion, and rearrangement. Therefore, the $S M N$ copies varied in different individuals and different populations (controls, SMA carriers, and patients) [5]. However, it is unclear how the $S M N$ copy number changes between the generations. In this study, we analyzed the copy number in SMN1 and SMN2 from 227 SMA core families to define the relationship between $S M N 1$ and $S M N 2$ and explore the variability in $S M N$ copy number within the generations.

\section{Materials and methods}

\section{Subjects}

In brief, 227 SMA core families registered in the "National rare diseases registry system of China" (https://www.nrdrs.org.cn) 
from the Department of Medical Genetics of Capital Institute of Pediatrics were recruited for the study, including 227 unrelated SMA children and their parents $(n=454)$ between March 2017 and July 2019. Molecular diagnosis was conducted for all SMA children. Genomic DNA was extracted from the peripheral blood using the EasyPure Blood Genomic DNA Kit (Transgen, Beijing, China). All participants signed informed consent, and this study was approved by the Ethics Committee of Capital Institute of Pediatrics.

\section{Quantitative analysis of SMN and SMN1 variant screening}

SMN1 and SMN2 copy numbers were determined by multiplex ligation-dependent probe amplification (MLPA) using the P060-B2 SMA kit (MRC-Holland, Amsterdam, Netherlands). For SMA children with heterozygous deletion in SMN1, Sanger sequencing strategy [6] was used for the subtle variant screening.

\section{Linkage analysis}

For the suspected ' $2+0$ ' carriers, further linkage analysis was conducted to define $S M N 1$ copy number on each allele. A total of 12 polymorphic markers (UHM (upstream haplotype marker) 2, UHM3, UHM4, UHM5, UHM7, UHM8; DHM (downstream haplotype marker) 1, DHM2, DHM4, DHM6, DHM7, and DHM8) within $2 \mathrm{Mb}$ of $S M N 2$ upstream and SMN1 downstream were used for linkage analysis, which was completed by Shanghai Chromysky Medical Research Co., Ltd.

\section{Statistical analyses}

The average copy numbers of SMN1 and SMN2 in two groups (e.g., SMA and carriers) or multiple groups (e.g., SMA I, II, and III) were compared using the $t$ test and ANOVA test, respectively. The rank correlation analysis was used to assess the association between the copy number of SMN1 and SMN2. Contingency table $\chi^{2}$ test was carried out to evaluate the distribution differences of SMA types among different $S M N 2$ copies. Statistical analyses were performed using the software package SPSS 23 for Window (SPSS Inc., Chicago, IL, USA). $P<0.05$ was considered to be statistically significant.

\section{Results}

The 227 SMA children included 64 patients with SMA type I, 101 patients with SMA type II, and 62 patients with SMA type III. The distribution of copy numbers of SMN1 and
Table 1 SMN copy numbers in 227 SMA families

\begin{tabular}{|c|c|c|}
\hline Items & Children; $N(\%)$ & Parents; $N(\%$ \\
\hline \multicolumn{3}{|l|}{ SMN1 copy number } \\
\hline 0 & $210(92.5)$ & 0 \\
\hline 1 & $17(7.5)$ & $411(90.5)$ \\
\hline 2 & 0 & $43(9.5)^{\mathrm{a}}$ \\
\hline Average $(\text { mean } \pm \text { SEM })^{b}$ & $0.07 \pm 0.018$ & $1.09 \pm 0.014$ \\
\hline \multicolumn{3}{|l|}{$S M N 2$ copy number } \\
\hline 1 & $1(0.4)$ & $68(15.0)$ \\
\hline 2 & $51(22.5)$ & $238(52.4)$ \\
\hline 3 & $169(74.4)$ & $144(31.7)$ \\
\hline 4 & $6(2.6)$ & $3(0.7)$ \\
\hline 5 & 0 & $1(0.2)$ \\
\hline Average $(\text { mean } \pm \text { SEM })^{b}$ & $2.79 \pm 0.032$ & $2.19 \pm 0.032$ \\
\hline
\end{tabular}

ancluding 17 parents with SMN1 subtle variant, 8 with ' $2+0$ ', genotype, and 18 suspected with ' $2+0$ ' genotype

${ }^{\mathrm{b}} P<0.001$ analyzed by $t$ test

SMN2 was shown in Table 1. In 227 SMA, 210 cases carried SMN1 homozygous deletions and 17 cases carried compound heterozygous variants in SMN1; 169 had three copies of $S M N 2$, which accounted for $74.4 \%$ of patients. In 454 SMA parents (obligate SMA carriers), as expected, 411 individuals had one copy of SMN1. However, 43 parents had two copies of SMN1, which included 17 parents with SMN1 subtle variant in one allele. The remaining 26 parents from 26 different families were suspected with the ' $2+0$ ' genotype, which was characterized by that one chromosome carried two copies of SMN1 in cis while the other chromosome carried none. Eight of them $(8 / 26 ; 30.77 \%)$ were willing to accept further family analysis. Combined SMNI copy number and linkage analyses indicated that the eight parents had two SMN1 copies on one allele, confirming the ' $2+0$ ' genotype.

In our study, the average copies of SMN1 in SMA were $0.07 \pm 0.018$ (mean \pm standard error mean, SEM), which were significantly lower than those in parents $(1.09 \pm 0.014$; $P<0.001)$. In contrast, the average copies of SMN2 in SMA were significantly higher than the parents $(2.79 \pm 0.032$ vs. $2.19 \pm 0.032 ; P<0.001)$. Among $227 \mathrm{SMA}$ and 454 parents, when the SMN1 copy number was 0,1 , and 2 , the average copies of $S M N 2$ were $2.82 \pm 0.031,2.24 \pm 0.033$, and $1.72 \pm$ $0.077(P<0.001)$, respectively. As seen in Fig. 1a, there was a negative correlation (Spearman $=-0.472, P<0.001$ ). Subsequently, we further analyzed the correlation between average SMN2 copies and SMN1 genotypes. When the $S M N 1$ genotype was ' $0+0$ ' $(S M N 1$ copy number $=0)$, ' $0+1^{\mathrm{D}}$ ' $($ SMN1 copy number $=1)$, ' $1+0$ ' (SMN1 copy number $=1),{ }^{\prime} 1+1^{\mathrm{D}}$ ' $(S M N 1$ copy number $=2)$ and ' $2+0$ ' $(S M N 1$ copy number $=2)$, the average copies of $S M N 2$ were $2.82 \pm 0.031,2.41 \pm 0.15,2.24 \pm 0.034,1.82 \pm 0.095$, and 


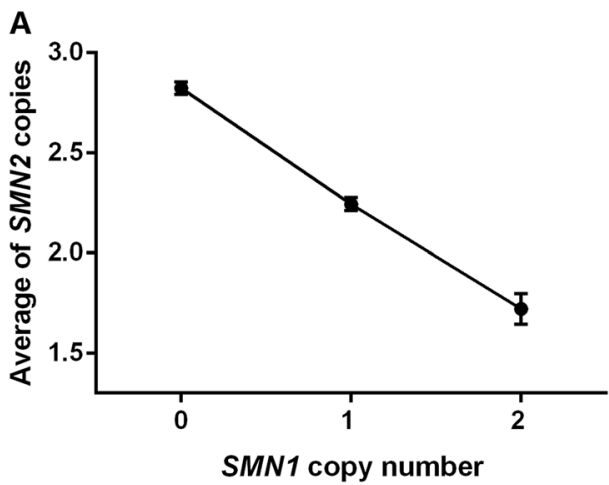

Fig. 1 The correlation between SMN in 227 SMA families. a The average of $S M N 2$ copies and SMN1 copies; b The average of SMN2 copies and SMN1 genotypes. The data were expressed as mean \pm SEM. The genotype of ' $0+0$ ' represents $S M N 1$ homozygous deletion,

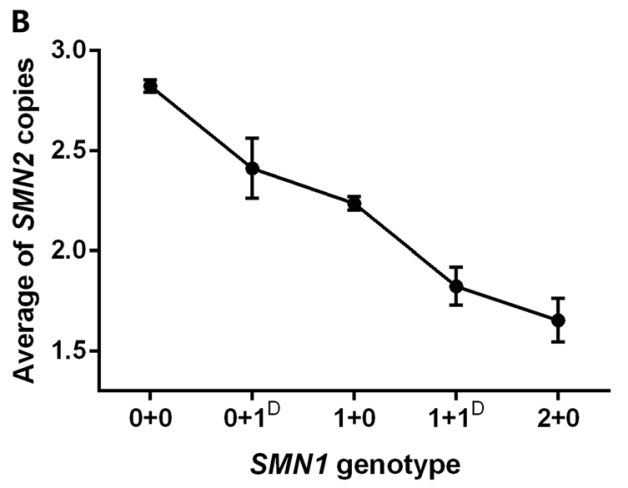

' $0+1^{\mathrm{D}}$ ' represented SMN1 compound heterozygous mutation, ' $1+0$ ' represented classical carrier, ' $1+1^{\mathrm{D}}$ ' represented one wild-type allele and the other allele with a mutation on each chromosome, and ' $2+0$ ' represented two SMN1 alleles on the same chromosome
Fig. 2 The average copies of $S M N$ in the parent's generation and SMA children among SMA types. a The average of SMN1 copies. b The average of SMN2 copies. The data were expressed as mean \pm SEM. The copies in the parent's generation was the average of SMN copies from father and mother in each family ((father + mother $) / 2) . * P<$ 0.001 compared with type I
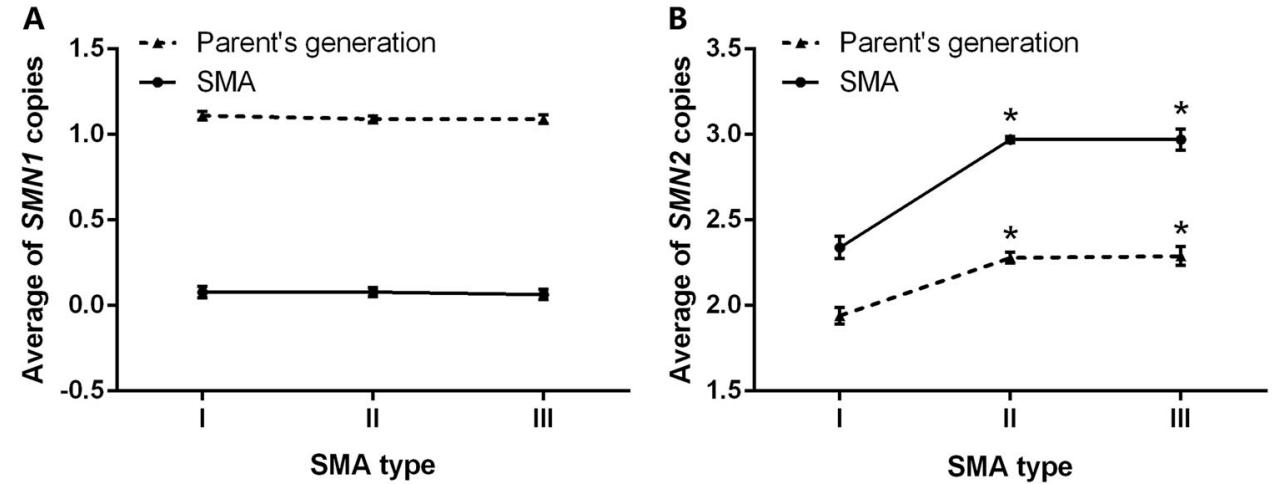

$1.65 \pm 0.11(P<0.001)$, respectively (Fig. 1b). Similarly, accompanied by the increased number of SMN1 copies, the average copy number of SMN2 decreased gradually (Spearman $=-0.471 ; P<0.001)$. Interestingly, for individuals with $S M N 1$ genotype ' $0+1^{\mathrm{D}}$ ' and ' $1+0$ ', although the copy number of $S M N 1$ was both one, there was a trend that the SMN2 average copy number of the former was higher than that of the latter $(2.41 \pm 0.15$ vs. $2.24 \pm 0.034)$; the same trend was found in individuals with $S M N 1$ genotype of ' $1+1^{\mathrm{D}}$ ' and ' $2+0$ ' $(1.82 \pm 0.095$ vs. $1.65 \pm$ 0.11 ), although they all carried the same two copies of SMN1.

If the variation of $S M N$ copies comes from inheritance, the $S M N$ copy number of children is determined by the $S M N$ copy number of their father and mother. Therefore, we used the average $S M N$ copies from the parents in each SMA family, namely, (1/2 SMN1 copies from father +1/2 SMN1 copies from mother) and (1/2 SMN2 copies from father $+1 / 2$ SMN2 copies from mother) to represent the copy number in the parent's generation, and then we compared $S M N$ copy numbers between the parent's generation and SMA children to explore whether the variation of SMN copy number originated from inheritance or de novo in generations. The average number of SMN1 copies was significantly higher in the parent's generation than those in SMA regardless of SMA types (all $P<0.001$ ) (Fig. 2a). By contrast, the average number of SMN2 copies was lower in the parent's generation than those of SMA (all $P<0.001$ ) (Fig. 2b). Moreover, the average $S M N 1$ copy number in the parent's generation whose SMA offspring was type I, type II, and type III was $1.11 \pm$ $0.026,1.09 \pm 0.019$, and $1.09 \pm 0.024$, respectively, and there was no difference among the three types of SMA $(P=0.801)$ (Fig. 2a). However, type II and type III SMA parent's generation had more copies of SMN2 than type I SMA parent's generation (both $P<0.001$ ) (Fig. 2b). Similarly, no difference was found in 227 SMA children carrying the average of SMN1 copies of $0.078 \pm 0.034$ for type I, $0.079 \pm 0.027$ for type II and $0.065 \pm 0.031$ for type III $(P=0.936)$ (Fig. 2a) and more SMN2 copies were observed in type II $(2.97 \pm$ $0.017)$ and III $(2.97 \pm 0.061)$ than type I $(2.34 \pm 0.064)$ SMA (both $P<0.001$ ) (Fig. 2b). The correlation analysis showed there was a significantly positive correlation for $S M N$ copies between the parent's generation and SMA children (Spearman $=0.589 ; P<0.001$ for $S M N 1$ and Spearman $=0.486$; $P<0.001$ for SMN2) (Supplementary Table 3). These data mentioned above suggested that the distribution trend of SMN1 and SMN2 copy number was consistent between the parent's generation and SMA children. 
As the most important phenotype-modified gene, SMN2, the copy number of this gene was negatively related to the severity of SMA. In order to determine whether the SMN2 copy number of the parent's generation could predict the SMA severity of the offspring, we calculated the proportion of the offspring suffering from heavy SMA (type I) and relatively light SMA (type II / III) when the SMN2 copy number of the parent's generation was fixed. For example, when the SMN2 copy number was one in the parent's generation, $75 \%$ of their SMA offspring had type I and $25 \%$ of them had type II/III. However, when the parent's generation carried three copies of SMN2, all of their SMA offspring were type II/III (Fig. 3). The distribution of type I and type II/III was significantly different in different SMN2 copies $\left(\chi^{2}=37.061, P<0.001\right)$.

\section{Discussion}

In this study, we analyzed the copy numbers of $S M N$ from 227 SMA children and 454 their parents using MLPA combined with Sanger sequencing strategy. The results showed that $92.5 \%$ of SMA children had zero copy of SMN1 and $7.5 \%$ had one copy of SMN1 with the SMN2 copies ranged from one to four. The proportion of SMN1 compound heterozygous variants $(7.5 \%)$ in this study was higher than the previously reported $\sim 5 \%$ [4]. In the parents, $90.5 \%$ had one copy of SMN1 and $9.5 \%$ had two copies of $S M N 1$ with the SMN2 copies ranged from one to five. For the parents with two SMN1 copies, except for 17 parents carry one normal allele and a pathogenic variant in the other allele, eight of 26 parents were confirmed ' $2+0$ ' genotype in SMN1 by dosage and linkage analysis. The remaining 18 parents with two copies of SMN1 could not be distinguished the ' $2+0$ ' carriers or noncarriers with ' $1+1$ ' genotype. However, considered their SMA children and spouses were all homozygous deletions and heterozygous deletion in $S M N 1$, respectively, the ' $2+0$ ' genotype was the most likely.

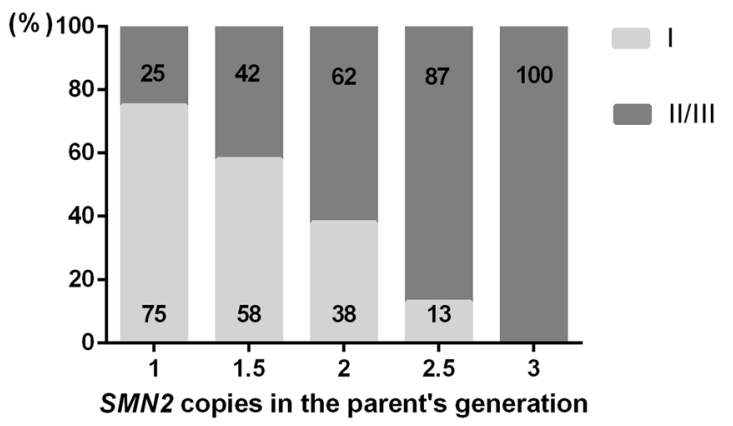

Fig. 3 SMN2 copies in the parent's generation and the disease severity of their SMA children. SMN2 copy number was the average SMN2 copies from parents in each family $(($ father + mother $) / 2)$
Reduction in copy number of SMN1 can be caused by either gene conversion or deletion [7]. There was bidirectional gene conversion between SMN1 and SMN2 in different population groups [8]. As early as 1997, the physical evidence was presented for conversion of SMN1-to-SMN2 in SMA patients and carriers $[9,10]$. However, the gene conversion in the opposite direction, SMN2-to-SMN1 was found in general population [10]. As shown in Fig. 1, in our 227 SMA families, the SMN1 copies were negatively correlated with the SMN2 copies, suggesting that there might be gene conversion. Subsequently, we analyzed the relationship between SMN2 copies and SMN1 genotype and found that with the increase in SMN1 copies, SMN2 copy number gradually decreased, presenting an inverse correlation between them. Moreover, there was a notable tendency for the individuals with function impaired alleles in SMN1 to have more SMN2 copies, compared with the alleles with nonimpaired function: $0+1^{\mathrm{D}}$, $(2.41 \pm 0.15)$ vs. ' $1+0$ ' $(2.24 \pm 0.034)($ Fig. $1 b)$ and ' $1+1^{\mathrm{D}}$, $(1.82 \pm 0.095)$ vs. ' $1+1$ ' $(1.56 \pm 0.076)$ (Fig. 1b; Supplementary Table 1), although the copy number of SMN1 was the same. This suggested that the dose compensation of SMN2 was related to not only the SMN1 copies but also the $S M N 1$ function. When the copy number of $S M N 1$ is the same, the individual with severely damaged function in SMN1 allele tends to have more SMN2 copies to compensate the deficiency of SMN protein. Besides the pathogenic variant will affect the function of $S M N 1$, some studies also had shown that gene conversion was common in mild SMA, while deletion was relatively common in severe SMA, indicating that the function of SMN1 might differ as a result of conversion and deletion [11].

SMN1 and SMN2 are all located in a region of chromosome $5 q 13$, which includes many homologous and mirroraligned genes. The complexity of this region has made it unstable and prone to deletion or conversion. We analyzed the copy number of SMN1 and SMN2 in SMA children and their parents' generation to determine whether the variation in $S M N$ copies resulted from inheritance or de novo. The results showed that the variation trend of SMN1 and SMN2 copy number was the same in SMA children and their parents' generation regardless the SMA types. And there was a positive correlation for SMN copies between these two generations.

In addition, we also analyzed the copy number of $S M N$ in 27 phenylketonuria (PKU) core families, a common genetic metabolic disease, which caused by variants in $P A H$. The results showed that the distribution of both the copy number of SMN1 and SMN2 had no significant difference between the PKU children and their parents $(P=0.607$ for $S M N 1$ and $P=0.326$ for $S M N 2$; contingency table $\chi^{2}$ test) (Supplementary Table 2). And there was a positive correlation between PKU children and their parents' generation both in the copy number $S M N 1$ and SMN2 (Spearman $=0.75$, 
$P<0.001$ for $S M N 1$; Spearman $=0.696, P<0.001$ for $S M N 2)$. All the results from SMA families and PKU families suggested that the variation of SMN copy number was largely inherited and could be steadily transmitted. Therefore, we can infer that the SMN2 copy number in the parent's generation has an effect on those of SMA children and then determine the disease severity to some extent.

Molecular characteristics of the SMN region determined that an individual might have gene deletion or conversion at a certain time. Under the founder effect, two populations with special variation in $S M N$ copy number, SMA carriers and SMA patients, were gradually formed. This phenomenon had both occasionality and universality. Occasionality may be the initiating factor of gene deletion or conversion. Some studies have shown that unequal crossing-over of SMN1 loci in meiosis might be the cause [12]; however, a certain universality may be due to the fact that the population carriers and morbidity of SMA are not rare.

In conclusion, our study showed an inverse correlation of the copy number between SMN1 and SMN2 in SMA children and their parents, suggesting the $S M N 1$-to-SMN2 gene conversion; the variation of $S M N$ copy number was resulted from inheritance and therefore, the disease severity of the SMA children was determined by their parent's generation to some extent.

Acknowledgements This study was supported by The National Key Research and Development Program of China (2016YFC0901505), CAMS Initiative for Innovative Medicine (CAMS-I2M-1-008), the China Postdoctoral Science Foundation (2018M630108), National Natural Science Foundation of China (81500979), Beijing Natural Science Foundation $(5163028,7172039)$. And we want to thank the patients and their parents for their collaboration in this investigation.

\section{Compliance with ethical standards}

Conflict of interest The authors declare that they have no conflict of interest.

Publisher's note Springer Nature remains neutral with regard to jurisdictional claims in published maps and institutional affiliations.

\section{References}

1. Ogino S, Leonard DG, Rennert H, Ewens WJ, Wilson RB. Genetic risk assessment in carrier testing for spinal muscular atrophy. Am J Med Genet. 2002;110:301-7.

2. Sugarman EA, Nagan N, Zhu H, Akmaev VR, Zhou Z, Rohlfs EM, et al. Pan-ethnic carrier screening and prenatal diagnosis for spinal muscular atrophy: clinical laboratory analysis of $>72$, 400 specimens. Eur J Hum. 2012;20:27-32.

3. Wirth B, Schmidt T, Hahnen E, Rudnik-Schöneborn S, Krawczak M, Muller-Myhsok B, et al. De novo rearrangements found in 2\% of index patients with spinal muscular atrophy: mutational mechanisms, parental origin, mutation rate, and implications for genetic counseling. Am J Hum Genet. 1997;61:1102-11.

4. Wirth B. An update of the mutation spectrum of the survival motor neuron gene (SMN1) in autosomal recessive spinal muscular atrophy (SMA). Hum Mutat 2000;15:228-37.

5. Sheng-Yuan Z, Xiong F, Chen YJ, Yan TZ, Zeng J, Li L, et al. Molecular characterization of SMN copy number derived from carrier screening and from core families with SMA in a Chinese population. Eur J Hum Genet. 2010;18:978-84.

6. Cao YY, Zhang WH, Qu YJ, Bai JL, Jin YW, Wang H, et al. Diagnosis of spinal muscular atrophy a simple method for quantifying the relative amount of SMN12 using Sanger DNA sequencing. Chin Med J. 2018;131:2921-9.

7. Burghes AH. When is a deletion not a deletion? When it is converted. Am J Hum Genet. 1997;61:9-15.

8. Chen TH, Tzeng CC, Wang CC, Wu SM, Chang JG, Yang SN, et al. Identification of bidirectional gene conversion between SMN1 and SMN2 by simultaneous analysis of SMN dosage and hybrid genes in a Chinese population. J Neurol Sci. 2011;308:83-7.

9. Campbell L, Potter A, Ignatius J, Dubowitz V, Davies K. Genomic variation and gene conversion in spinal muscular atrophy: implications for disease process and clinical phenotype. Am J Hum Genet. 1997;61:40-50.

10. Ogino S, Gao S, Leonard DG, Paessler M, Wilson RB. Inverse correlation between SMN1 and SMN2 copy number: evidence for gene conversion from SMN2 to SMN1. Eur J Hum Genet. 2003;11:275-7.

11. McAndrew PE, Parsons DW, Simard LR, Rochette C, Ray PN, Mendell JR, et al. Identification of proximal spinal muscular atrophy carriers and patients by analysis of SMNT and SMNC gene copy number. Am J Hum Genet. 1997;60:1411-22.

12. Cusin V, Clermont O, Gérard B, Chantereau D, Elion J. Prevalence of SMN1 deletion and duplication in carrier and normal populations: implication for genetic counseling. J Med Genet. 2003;40:e39. 